\title{
X-Ray Searches for Axions from Super Star Clusters
}

\author{
Christopher Dessert $\oplus^{1,2,3}$ Joshua W. Foster, ${ }^{1,2,3}$ and Benjamin R. Safdi® ${ }^{1,2,3}$ \\ ${ }^{1}$ Leinweber Center for Theoretical Physics, Department of Physics, University of Michigan, Ann Arbor, Michigan 48109, USA \\ ${ }^{2}$ Berkeley Center for Theoretical Physics, University of California, Berkeley, California 94720, USA \\ ${ }^{3}$ Theoretical Physics Group, Lawrence Berkeley National Laboratory, Berkeley, California 94720, USA
}

(Received 12 August 2020; revised 13 November 2020; accepted 23 November 2020; published 22 December 2020)

\begin{abstract}
Axions may be produced in abundance inside stellar cores and then convert into observable $\mathrm{x}$ rays in the Galactic magnetic fields. We focus on the Quintuplet and Westerlund 1 super star clusters, which host large numbers of hot, young stars including Wolf-Rayet stars; these stars produce axions efficiently through the axion-photon coupling. We use Galactic magnetic field models to calculate the expected x-ray flux locally from axions emitted from these clusters. We then combine the axion model predictions with archival Nuclear Spectroscopic Telescope Array (NuSTAR) data from 10-80 keV to search for evidence of axions. We find no significant evidence for axions and constrain the axion-photon coupling $g_{\text {arr }} \lesssim 3.6 \times$ $10^{-12} \mathrm{GeV}^{-1}$ for masses $m_{a} \lesssim 5 \times 10^{-11} \mathrm{eV}$ at $95 \%$ confidence.
\end{abstract}

DOI: 10.1103/PhysRevLett.125.261102

Ultralight axionlike particles that couple weakly to ordinary matter are natural extensions to the standard model. For example, string compactifications often predict large numbers of such pseudoscalar particles that interact with the standard model predominantly through dimensionfive operators [1,2]. If an axion couples to quantum chromodynamics (QCD) then it may also solve the strong $C P$ problem [3-6]; in this work we refer to both the QCD axion and axionlike particles as axions.

Axions may interact electromagnetically through the operator $\mathcal{L}=-g_{\text {ary }} a F_{\mu \nu} \tilde{F}^{\mu \nu} / 4$, where $a$ is the axion field, $F$ is the electromagnetic field-strength tensor, with $\tilde{F}$ its Hodge dual, and $g_{a \gamma \gamma}$ is the dimensionful coupling constant of axions to photons. This operator allows both the production of axions in stellar plasmas through the Primakoff process $[7,8]$ and the conversion of axions to photons in the presence of static external magnetic fields. Strong constraints on $g_{\text {ary }}$ for low-mass axions come from the CERN Axion Solar Telescope (CAST) experiment [9], which searches for axions produced in the Solar plasma that free stream to Earth and then convert to $\mathrm{x}$ rays in the magnetic field of the

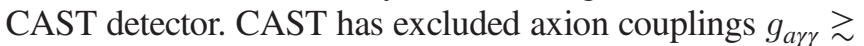
$6.6 \times 10^{-11} \mathrm{GeV}^{-1}$ for axion masses $m_{a} \lesssim 0.02 \mathrm{eV}$ at $95 \%$ confidence [9]. Primakoff axion production also opens a new pathway by which stars may cool, and strong imits $\left(g_{\text {ary }} \lesssim 6.6 \times 10^{-11} \mathrm{GeV}^{-1}\right.$ at $95 \%$ confidence for $m_{a} \lesssim \mathrm{keV}$ ) are derived from observations of the horizontal

Published by the American Physical Society under the terms of the Creative Commons Attribution 4.0 International license. Further distribution of this work must maintain attribution to the author(s) and the published article's title, journal citation, and DOI. Funded by SCOAP ${ }^{3}$. branch $(\mathrm{HB})$ star lifetime, which would be modified in the presence of axion cooling [10].

In this work, we produce some of the strongest constraints to date on $g_{\text {ary }}$ for $m_{a} \lesssim 10^{-9} \mathrm{eV}$ through x-ray observations with the Nuclear Spectroscopic Telescope Array (NuSTAR) telescope [11] of super star clusters (SSCs). The SSCs contain large numbers of hot, young, and massive stars, such as Wolf-Rayet (WR) stars. We show that these stars, and as a result the SSCs, are highly efficient at producing axions with energies $\sim 10-100 \mathrm{keV}$ through the Primakoff process. These axions may then convert into photons in the Galactic magnetic fields, leading to signatures observable with space-based $\mathrm{x}$-ray telescopes such as NuSTAR. We analyze archival NuSTAR data from the Quintuplet SSC near the Galactic Center (GC) along with the nearby Westerlund 1 (Wd1) cluster and constrain $g_{\text {ary }} \lesssim 3.6 \times 10^{-12} \mathrm{GeV}^{-1}$ at $95 \%$ confidence for $m_{a} \lesssim 5 \times 10^{-11} \mathrm{eV}$. In Fig. 1 we show the locations of the stars within the Quintuplet cluster that are considered in this work on top of the backgroundsubtracted NuSTAR counts, from $10-80 \mathrm{keV}$, with the point-spread function (PSF) of NuSTAR also indicated. In the Supplemental Material [12] we show that observations of the Arches SSC yield similar but slightly weaker limits.

Our work builds upon significant previous efforts to use stars as laboratories to search for axions. Some of the strongest constraints on the axion-matter couplings, for example, come from examining how $\mathrm{HB}$, white dwarf (WD), red giant, and neutron star (NS) cooling would be affected by an axion [10,20-28]. When the stars have large magnetic fields, as is the case for WDs and NSs, the axions can be converted to $\mathrm{x}$ rays in the stellar magnetospheres [29-32]. Intriguingly, in Refs. [31,32] observations of the Magnificent Seven nearby isolated NSs found evidence for 


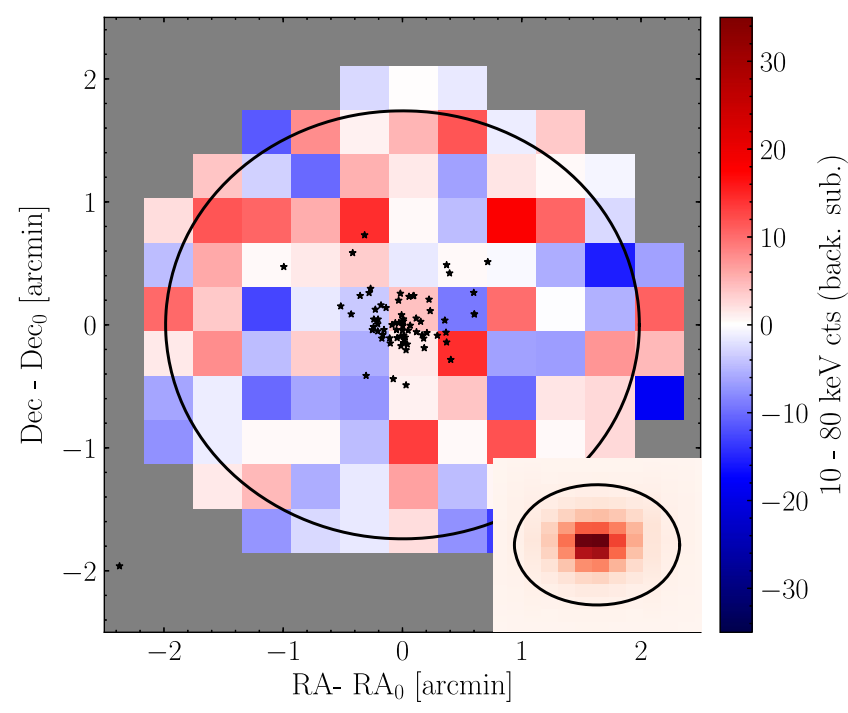

FIG. 1. The stacked and pixelated background-subtracted count data $(10-80 \mathrm{keV})$ from the NuSTAR observations of the Quintuplet SSC. The locations of the stars are indicated in black, while the $90 \%$ energy containment region for emission associated with the SSC is indicated by the black circle, accounting for the NuSTAR point spread function (PSF). $\mathrm{RA}_{0}$ and $\mathrm{DEC}_{0}$ denote the locations of the cluster center. We find no evidence for axioninduced emission from this SSC, which would follow the spatial counts template illustrated in the inset panel.

a hard x-ray excess consistent with the expected axion spectrum from nucleon bremsstrahlung. This work extends these efforts by allowing the axions to convert to $\mathrm{x}$ rays not just in the stellar magnetic fields but also in the Galactic magnetic fields [33-35].

Axion production in SSCs.-During helium burning, particularly massive stars may undergo considerable mass loss, especially through either rotation or binary interaction, which can begin to peel away the hydrogen envelope, revealing the hot layers underneath and reversing the cooling trend. Stars undergoing this process are known as WR stars, and these stars are the most important in our analyses. If the star has a small $(<40 \%$ abundance) remaining hydrogen envelope, it is classified as a WNh star; at $<5 \%$ hydrogen abundance it is classified as a WN star; otherwise, it is classified as WC or WO, which indicates the presence of $>2 \%$ carbon, and oxygen, respectively, in the atmosphere.

Axions are produced through the photon coupling $g_{a \gamma \gamma}$ in the high-mass stars in SSCs through the Primakoff process $\gamma+\left(e^{-}, Z\right) \rightarrow a+\left(e^{-}, Z\right)$. This process converts a stellar photon to an axion in the screened electromagnetic field of the nucleons and electrons. The massive stars are high temperature and low density and therefore form nonrelativistic nondegenerate plasmas. The Primakoff emission rate was calculated in Refs. $[8,36]$ as a function of temperature, density, and composition, and is described in detail in the Supplemental Material [12].
To compute the axion luminosity in a given star, we use the stellar evolution code Modules for Experiments in Stellar Astrophysics (MESA) [37,38] to find, at any particular time in the stellar evolution, radial profiles of temperature, density, and composition. The simulation states are specified by an initial metallicity $Z$, an initial stellar mass, an initial rotation velocity, and an age. The initial metallicity is taken to be constant for all stars. In the Supplemental Material [12] we show that the Quintuplet and Arches clusters, which are both near the GC, are likely to have initial metallicities in the range $Z \in(0.018,0.035)$, consistent with the conclusions of previous works which place the initial metallicities of these clusters near solar (solar metallicity is $Z \approx 0.02$ ) $[39,40]$. Note that higher metallicities generally lead to the stars entering the WR classifications sooner, when their cores are cooler. Rotation may also cause certain massive stars to be classified as WR stars at younger ages. We model the initial rotation distribution as a Gaussian distribution with mean $\mu_{\text {rot }}$ and standard deviation $\sigma_{\text {rot }}$ for non-negative rotation speeds [41,42]. References [41,42] found $\mu_{\text {rot }} \approx 100$ and $\sigma_{\text {rot }} \approx 140 \mathrm{~km} / \mathrm{s}$, but to assess systematic uncertainties we vary $\mu_{\text {rot }}$ between 50 and $150 \mathrm{~km} / \mathrm{s}$ [41].

We draw initial stellar velocities from the velocity distribution described above (from 0 to $500 \mathrm{~km} / \mathrm{s}$ ) and initial stellar masses from the Kroupa initial mass function [43] (from 15 to $200 M_{\odot}$ ). We use MESA to evolve the stars from pre-main sequence (pre-MS) - before core hydrogen ignition-to near supernova. At each time step we assign each stellar model a spectroscopic classification using the definitions in Refs. $[44,45]$. We then construct an ensemble of models for each spectroscopic classification by joining together the results of the different simulations that result in the same classification for stellar ages within the age range for star formation in the cluster; for Quintuplet, this age range is between 3.0 and 3.6 Myr [46]. Note that each simulation generally provides multiple representative models, taken at different time steps. In total we compute $10^{5}$ models per stellar classification.

Quintuplet hosts 71 stars of masses $\gtrsim 50 M_{\odot}$, with a substantial WR cohort [46]. In particular it has 14 $\mathrm{WC}+\mathrm{WN}$ stars, and we find that these stars dominate the predicted axion flux. For example, at $g_{a \gamma \gamma}=$ $10^{-12} \mathrm{GeV}^{-1}$ we compute that the total axion luminosity from the SSC (with $Z=0.035$ and $\mu_{\text {rot }}=150 \mathrm{~km} / \mathrm{s}$ ) is $2.1_{-0.4}^{+0.7} \times 10^{35} \mathrm{erg} / \mathrm{s}$, with $\mathrm{WC}+\mathrm{WN}$ stars contributing $\sim 70 \%$ of that flux. Note that the uncertainties arise from performing multiple (500) draws of the stars from our ensembles of representative models. In the 10-80 keV energy range relevant for NuSTAR the total luminosity is $1.7_{-0.3}^{+0.4} \times 10^{35} \mathrm{erg} / \mathrm{s}$. We take $Z=0.035$ and $\mu_{\text {rot }}=$ $150 \mathrm{~km} / \mathrm{s}$ because these choices lead to the most conservative limits. For example, taking the metallicity at the lower end of our range $(Z=0.018)$ along with $\mu_{\text {rot }}=100 \mathrm{~km} / \mathrm{s}$ the predicted $10-80 \mathrm{keV}$ flux increases by 
$\sim 60 \%$. At fixed $Z=0.035$ changing $\mu_{\text {rot }}$ from 150 to $100 \mathrm{~km} / \mathrm{s}$ increases the total luminosity (over all energies) by $\sim 10 \%$, though the luminosity in the $10-80 \mathrm{keV}$ range is virtually unaffected.

The $\mathrm{Wd} 1$ computations proceed similarly. $\mathrm{Wd} 1$ is measured from parallax to be a distance $d \in(2.2,4.8) \mathrm{kpc}$ from the Sun [47], accounting for both statistical and systematic uncertainties [48]. Wd1 is estimated to have an age between 4.5 and 7.1 Myr from isochrone fitting, which we have broadened appropriately from Ref. [49] accounting for expanded distance uncertainties. In our fiducial analysis we simulate the stars in $\mathrm{Wd} 1$ for initial metallicity $Z=0.035$ and $\mu_{\text {rot }}=150 \mathrm{~km} / \mathrm{s}$ as this leads to the most conservative flux predictions, even though it is likely that the metallicity is closer to solar for $\mathrm{Wd} 1$ [50], in which cases the fluxes are larger by almost a factor of 2 (see the Supplemental Material [12]). We model 153 stars in Wd1 [49], but the axion flux is predominantly produced by the 8 WC and $14 \mathrm{WN}$ stars. In total we find that the $10-80 \mathrm{keV}$ luminosity, for $g_{\text {ary }}=10^{-12} \mathrm{GeV}$, is $9.02_{-1.1}^{+1.2} \times 10^{35} \mathrm{erg} / \mathrm{s}$, which is $\sim 5$ times larger than that from Quintuplet.

Axion conversion in Galactic fields.-The axions produced within the SSCs may convert to $\mathrm{x}$ rays in the Galactic magnetic fields. The axion Lagrangian term $\mathcal{L}=g_{\text {ary }} a \mathbf{E} \cdot \mathbf{B}$, written in terms of electric and magnetic fields $\mathbf{E}$ and $\mathbf{B}$, causes an incoming axion state to rotate into a polarized electromagnetic wave in the presence of an external magnetic field (see, e.g., Ref. [51]). The conversion probability $p_{a \rightarrow \gamma}$ depends on the transverse magnetic field, the axion mass $m_{a}$, and the plasma frequency $\omega_{\mathrm{pl}} \approx 3.7 \times 10^{-12}\left(n_{e} / 10^{-2} \mathrm{~cm}^{-3}\right)^{-1 / 2} \mathrm{eV}$, with $n_{e}$ the free-electron density (see the Supplemental Material [12] for an explicit formula). Note that hydrogen absorption towards all of our targets is negligible, being at most 5\% in the $15-20 \mathrm{keV}$ bin of the Quintuplet analysis [52].

To compute the energy-dependent conversion probabilities $p_{a \rightarrow \gamma}$ for our targets we need to know the magnetic field profiles and electron density distributions along the lines of sight. For our fiducial analysis we use the regular components of the JF12 Galactic magnetic field model [53,54] and the YMW16 electron density model [55] (though in the Supplemental Material [12] we show that the ne2001 [56] model gives similar results), though the JF12 model does not cover the inner kpc of the Galaxy. Outside of the inner kpc the conversion probability for Quintuplet is dominated by the out-of-plane ( $X$-field) component in the JF12 model. We conservatively assume that the magnitude of the vertical magnetic field within the inner $\mathrm{kpc}$ is the same as the value at $1 \mathrm{kpc}\left(\left|B_{z}\right| \approx 3 \mu \mathrm{G}\right)$, as illustrated in Supplemental Material [12], Fig. S6. In our fiducial magnetic field model the conversion probability is $p_{a \rightarrow \gamma} \approx 2.4 \times 10^{-4}\left(7 \times 10^{-5}\right)$ for $g_{a \gamma \gamma}=10^{-12} \mathrm{GeV}^{-1}$ for axions produced in the Quintuplet SSC with $m_{a} \ll$ $10^{-11} \mathrm{eV}$ and $E=80 \mathrm{keV}(E=10 \mathrm{keV})$. Completely masking the inner kpc reduces these conversion probabilities to $p_{a \rightarrow \gamma} \approx 1.0 \times 10^{-4}\left(p_{a \rightarrow \gamma} \approx 3.2 \times 10^{-5}\right)$, for $E=80 \mathrm{keV}$ $(E=10 \mathrm{keV})$. On the other hand, changing the global magnetic field model to that presented in Ref. [57] (PTKN11), which has a larger in-plane component than the JF12 model but no out-of-plane component, leads to conversion probabilities at $E=80$ and $10 \mathrm{keV}$ of $p_{a \rightarrow \gamma} \approx$ $4.9 \times 10^{-4}$ and $p_{a \rightarrow \gamma} \approx 4.2 \times 10^{-5}$, respectively, with the inner kpc masked.

The magnetic field is likely larger than the assumed $3 \mu \mathrm{G}$ within the inner kpc. Note that the local interstellar magnetic field, as measured directly by the Voyager missions [58], indirectly by the Interstellar Boundary Explorer [59], inferred from polarization measurements of nearby stars [60], and inferred from pulsar dispersion measure and the rotation measure data [61], has magnitude $B \sim 3 \mu \mathrm{G}$, and all evidence points to the field rising significantly in the inner $\mathrm{kpc}$ [62]. For example, Ref. [63] bounded the magnetic field within the inner $400 \mathrm{pc}$ to be at least $50 \mu \mathrm{G}$, and more likely $100 \mu \mathrm{G}$ (but less than $\sim 400 \mu \mathrm{G}$ [64]), by studying nonthermal radio emission in the inner Galaxy. Localized features in the magnetic field in the inner kpc may also further enhance the conversion probability beyond what is accounted for here. For example, the line-of-sight to the Quintuplet cluster overlap with the GC radio arc nonthermal filament, which has a $\sim 3 \mathrm{mG}$ vertical field over a narrow filament of cross section $\sim(10 \mathrm{pc})^{2}$ (see, e.g., Ref. [65]). Accounting for the magnetic fields structures described above in the inner few hundred pc may enhance the conversion probabilities by over an order of magnitude relative to our fiducial scenario (see the Supplemental Material [12]).

When computing the conversion probabilities for Wd1 we need to account for the uncertain distance $d$ to the SSC (with the currently allowable range given above). In the JF12 model we find the minimum $p_{a \rightarrow \gamma} / d^{2}$ (for $m_{a} \ll 10^{-11} \mathrm{eV}$ ) is obtained for $d \approx 2.6 \mathrm{kpc}$, which is thus the value we take for our fiducial distance in order to be conservative. At this distance the conversion probability is $p_{a \rightarrow \gamma} \approx 2.4 \times 10^{-6}\left(p_{a \rightarrow \gamma} \approx 1.5 \times 10^{-6}\right)$ for $E=10 \mathrm{keV}$ $(E=80 \mathrm{keV})$, assuming $g_{\text {ary }}=10^{-12} \mathrm{GeV}^{-1}$ and $m_{a} \ll 10^{-11} \mathrm{eV}$. We note that the conversion probabilities are over 10 times larger in the PTKN11 model (see the Supplemental Material [12]), since there is destructive interference (for $d \approx 2.6 \mathrm{kpc}$ ) in the JF12 model towards Wd1. We do not account for turbulent fields in this analysis; inclusion of these fields may further increase the conversion probabilities for $\mathrm{Wd} 1$, although we leave this modeling for future work.

Data analysis.-We reduce and analyze $39 \mathrm{ks}$ of archival NuSTAR data from Quintuplet with observation ID 40010005001 . This observation was performed as part of the NuSTAR Hard X-ray Survey of the GC Region $[66,67]$. The NuSTAR data reduction was performed with the HEASoft software version 6.24 [68]. This process leads to a set of counts, exposure, and background maps for every 
energy bin and for each exposure (we use data from both Focal Plane Modules A and B). The astrometry of each exposure is calibrated independently using the precise location of the source 1E 1743.1-2843 [69], which is within the field of view. The background maps account for the cosmic x-ray background, reflected solar x rays, and instrumental backgrounds such as Compton-scattered gamma rays and detector and fluorescence emission lines [70]. We then stack and rebin the datasets to construct pixelated images in each of the energy bins. We use 14 5 -keV-wide energy bins between 10 and $80 \mathrm{keV}$. We label those images $d_{i}=\left\{c_{i}^{p}\right\}$, where $c_{i}^{p}$ stands for the observed counts in energy bin $i$ and pixel $p$. The pixelation used in our analysis is illustrated in Fig. 1.

For the Wd1 analysis we reduced Focal Plane Module A and $\mathrm{B}$ data totaling $138 \mathrm{ks}$ from observation IDs 80201050008,80201050006 , and 80201050002 . This set of observations was performed to observe outburst activity of the Wd1 magnetar CXOU J164710.2-45521 [71], which we mask at $0.5^{\prime}$ in our analysis. (The magnetar is around $1.5^{\prime}$ away from the cluster center.) Note that in Ref. [71] hard x-ray emission was only detected with the NuSTAR data from 3-8 keV from CXOU J164710.2-45521 - consistent with this, removing the magnetar mask does not affect our extracted spectrum for the SSC above $10 \mathrm{keV}$. We use the magnetar in order to perform astrometric calibration of each exposure independently. The Wd1 exposures suffer from ghost-ray contamination [72] from a nearby point source that is outside of the NuSTAR field of view at low energies (below $\sim 15 \mathrm{keV}$ ) [71]. (Ghost-ray contamination refer to those photons that reflect only a single time in the mirrors.) The ghost-ray contamination affects our ability to model the background below $15 \mathrm{keV}$ and so we remove the 10-15 keV energy bin from our analysis.

In each energy bin we perform a Poissonian template fit over the pixelated data to constrain the number of counts that may arise from the template associated with axion emission from the SSC. To construct the signal template we use a spherically symmetric approximation to the NuSTAR PSF [73] and we account for each of the stars in the SSC individually in terms of spatial location and expected flux, which generates a nonspherical and extended template. We label the set of signal templates by $S_{i}^{p}$. We search for emission associated with the signal templates by profiling over background emission. We use the set of background templates described above and constructed when reducing the data, which we label $B_{i}^{p}$.

Given the set of signal and background templates we construct a Poissonian likelihood in each energy bin:

$$
p_{i}\left(d_{i} \mid\left\{S_{i}, A_{B}\right\}\right)=\sum_{p} \frac{\left(\mu_{i}^{p}\right)^{c_{i}^{p}} e^{-\mu_{i}^{p}}}{c_{i}^{p} !},
$$

with $\mu_{i}^{p}=S_{i} S_{i}^{p}+A_{B} B_{i}^{p}$. We then construct the profile likelihood $p_{i}\left(d_{i} \mid\left\{S_{i}\right\}\right)$ by maximizing the log likelihood at

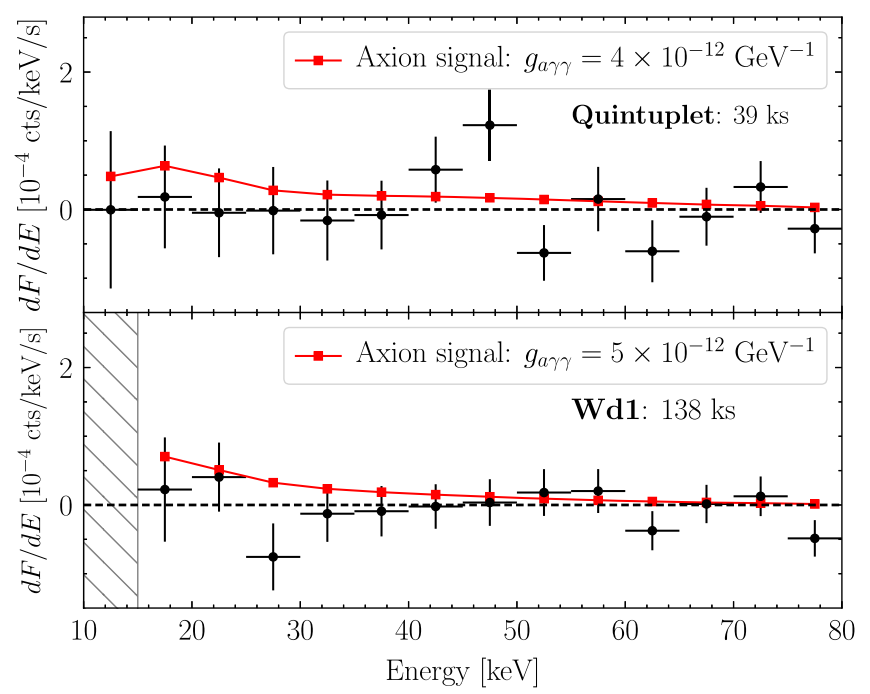

FIG. 2. The spectra associated with the axion-induced templates from the Quintuplet and Wd1 SSCs constructed from the NuSTAR data analyses, with best-fit points and $1 \sigma$ uncertainties indicated. In red we show the predicted spectra from an axion with $m_{a} \ll 10^{-11} \mathrm{eV}$ and indicated $g_{a \gamma \gamma}$. Note that for Wd1 we do not analyze the $10-15 \mathrm{keV}$ energy bin because of ghost-ray contamination.

each fixed $S_{i}$ over the nuisance parameter $A_{B}$. Note that when constructing the profile likelihood we use the region of interest (ROI) where we mask pixels further than 2.0' from the SSC center. The $90 \%$ containment radius of NuSTAR is $\sim 1.74$ ', independent of energy, as indicated in Fig. 1. We use a localized region around our source to minimize possible systematic biases from background mismodeling. However, as we show in the Supplemental Material [12] our final results are not strongly dependent on the choice of ROI. We also show in Ref. [12] that if we inject a synthetic axion signal into the real data and analyze the hybrid data, we correctly recover the simulated axion parameters.

The best-fit flux values and $1 \sigma$ uncertainties extracted from the profile likelihood procedure are illustrated in Fig. 2 for the Quintuplet and Wd1 datasets. We compare the spectral points to the axion model prediction to constrain the axion model. More precisely, we combine the profile likelihoods together from the individual energy bins to construct a joint likelihood that may be used to search for the presence of an axion signal: $p\left(d \mid\left\{m_{a}, g_{a \gamma \gamma}\right\}\right)=$ $\prod_{i} p_{i}\left[d_{i} \mid R_{i}\left(m_{a}, g_{a \gamma \gamma}\right)\right]$, where $R_{i}\left(m_{a}, g_{a \gamma \gamma}\right)$ denotes the predicted number of counts in the $i$ th energy bin given an axion-induced $\mathrm{x}$-ray spectrum with axion model parameters $\left\{m_{a}, g_{a \gamma \gamma}\right\}$. The values $R_{i}\left(m_{a}, g_{a \gamma \gamma}\right)$ are computed using the forward-modeling matrices constructed during the data reduction process.

In Fig. 3 we illustrate the 95\% power-constrained [74] upper limits on $g_{a \gamma \gamma}$ as a function of the axion mass $m_{a}$ found from our analyses. The joint limit (red in Fig. 3), 


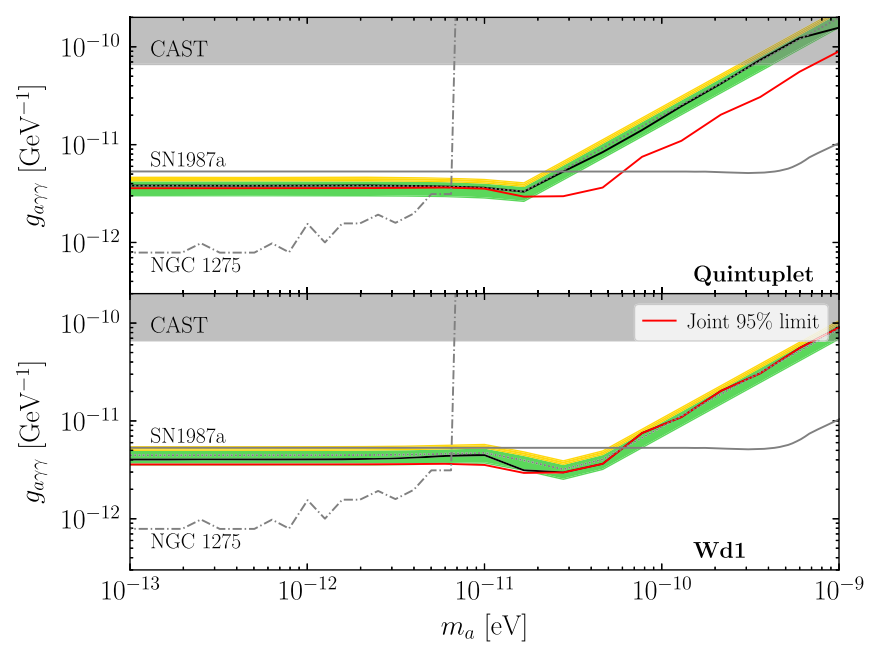

FIG. 3. The 95\% upper limits (black) on $g_{a \gamma \gamma}$ as a function of the axion mass from the Quintuplet and Wd1 data analyses. We compare the limits to the $1 \sigma$ (green band) and $2 \sigma$ (yellow band) expectations under the null hypothesis, along with the median expectations (dotted). The joint 95\% upper limit, combining Quintuplet and Wd1, is also indicated (expected joint limit not shown). At low masses our limits may be surpassed by those from searches for x-ray spectral modulations from NGC 1275 [76], though we caution that those limits have been called into question recently, as discussed further in the text [77].

combining the Quintuplet and Wd1 profile likelihoods, becomes $g_{\text {ary }} \lesssim 3.6 \times 10^{-12} \mathrm{GeV}^{-1}$ at low axion masses. At fixed $m_{a}$ the upper limits are constructed by analyzing the test statistic $q\left(g_{a \gamma \gamma} \mid m_{a}\right) \equiv 2 \ln p\left(d \mid\left\{m_{a}, g_{a \gamma \gamma}\right\}\right)-$ $2 \ln p\left(d \mid\left\{m_{a}, \bar{g}_{a \gamma \gamma}\right\}\right)$, where $\bar{g}_{\text {ary }}$ is the signal strength that maximizes the likelihood, allowing for the possibility of negative signal strengths as well. The $95 \%$ upper limit is given by the value $g_{a \gamma \gamma}>\bar{g}_{a \gamma \gamma}$ such that $q\left(g_{a \gamma \gamma} \mid m_{a}\right) \approx 2.71$ (see, e.g., Ref. [75]). The $1 \sigma$ and $2 \sigma$ expectations for the 95\% upper limits under the null hypothesis, constructed from the Asimov procedure [75], are also shown in Fig. 3. The evidence in favor of the axion model is $\sim 0.3 \sigma(0 \sigma)$ local significance at low masses for Quintuplet (Wd1).

We compare our upper limits with those found from the CAST experiment [9], the nonobservation of gamma rays from SN1987a [78] (see also Refs. [79-81] along with Ref. [82], who recently questioned the validity of these limits), and the NGC $1275 \mathrm{x}$-ray spectral modulation search [76]. It was recently pointed out, however, that the limits in Ref. [76] are highly dependent on the intracluster magnetic field models and could be orders of magnitude weaker, when accounting for both regular and turbulent fields [77]. The CAST limits are stronger than ours for $m_{a} \gtrsim 10^{-9} \mathrm{eV}$ and rely on less modeling assumptions, since CAST searches for axions produced in the Sun, though we have made conservative choices in our stellar modeling.

Discussion.-We present limits on the axion-photon coupling $g_{a \gamma \gamma}$ from a search with NuSTAR hard x-ray data for axions emitted from the hot, young stars within SSCs and converting to $\mathrm{x}$ rays in the Galactic magnetic fields. We find the strongest limits from analyses of data towards the Quintuplet and Wd1 clusters. Our limits represent some of the strongest and most robust limits to date on $g_{a \gamma \gamma}$ for lowmass axions. We find no evidence for axions. Promising targets for future analyses could be nearby supergiant stars, such as Betelgeuse [33,83], or young NSs such as Cas A.

Figures and supplementary data are provided in Ref. [84].

We thank Fred Adams, Malte Buschmann, Roland Crocker, Ralph Eatough, Glennys Farrar, Katia Ferrière, Andrew Long, Kerstin Perez, and Nick Rodd for helpful comments and discussions. This work was supported in part by the DOE Early Career Grant No. DESC0019225 and through computational resources and services provided by Advanced Research Computing at the University of Michigan, Ann Arbor.

[1] P. Svrcek and E. Witten, Axions in string theory, J. High Energy Phys. 06 (2006) 051.

[2] A. Arvanitaki, S. Dimopoulos, S. Dubovsky, N. Kaloper, and J. March-Russell, String axiverse, Phys. Rev. D 81, 123530 (2010).

[3] R. D. Peccei and H. R. Quinn, Constraints imposed by $C P$ conservation in the presence of instantons, Phys. Rev. D 16, 1791 (1977).

[4] R. D. Peccei and H. R. Quinn, $C P$ Conservation in the Presence of Instantons, Phys. Rev. Lett. 38, 1440 (1977).

[5] S. Weinberg, A New Light Boson?, Phys. Rev. Lett. 40, 223 (1978).

[6] F. Wilczek, Problem of Strong P and T Invariance in the Presence of Instantons, Phys. Rev. Lett. 40, 279 (1978).

[7] H. Primakoff, Photoproduction of neutral mesons in nuclear electric fields and the mean life of the neutral meson, Phys. Rev. 81, 899 (1951).

[8] G. G. Raffelt, Astrophysical axion bounds diminished by screening effects, Phys. Rev. D 33, 897 (1986).

[9] V. Anastassopoulos et al. (CAST Collaboration), New CAST limit on the axion-photon interaction, Nat. Phys. 13, 584 (2017).

[10] A. Ayala, I. Domínguez, M. Giannotti, A. Mirizzi, and O. Straniero, Revisiting the Bound on Axion-Photon Coupling from Globular Clusters, Phys. Rev. Lett. 113, 191302 (2014).

[11] F. A. Harrison et al., The nuclear spectroscopic telescope array (NuSTAR) high-energy X-ray mission, Astrophys. J. 770, 103 (2013).

[12] See Supplemental Material at http://link.aps.org/ supplemental/10.1103/PhysRevLett.125.261102 contains additional results and explanations of our methods that clarify and support the results presented in the main Letter. We present further details regarding the data analyses, simulations, and calculations, show additional results beyond those presented in the main Letter, and provide results of an auxiliary analysis used to derive the metallicity range considered in this work, which includes Refs. [13-19]. 
[13] E. Glebbeek, E. Gaburov, S. E. de Mink, O. R. Pols, and S. F.P. Zwart, The evolution of runaway stellar collision products, Astron. Astrophys. 497, 255 (2009).

[14] G. G. Raffelt, Astrophysical methods to constrain axions and other novel particle phenomena, Phys. Rep. 198, 1 (1990).

[15] F. Yusef-Zadeh, The Origin of the Galactic center nonthermal radio filaments: Young stellar clusters, Astrophys. J. 598, 325 (2003).

[16] J. S. Clark, M. E. Lohr, L. R. Patrick, and F. Najarro, The arches cluster revisited. III. An addendum to the stellar census, Astron. Astrophys. 623, A84 (2019).

[17] R. Krivonos, M. Clavel, J. Hong, K. Mori, G. Ponti, J. Poutanen, F. Rahoui, J. Tomsick, and S. Tsygankov, NuSTAR and XMM-Newton observations of the Arches cluster in 2015: Fading hard X-ray emission from the molecular cloud, Mon. Not. R. Astron. Soc. 468, 2822 (2017).

[18] R. A. Krivonos et al., First hard X-ray detection of the nonthermal emission around the Arches cluster: Morphology and spectral studies with NuSTAR, Astrophys. J. 781, 107 (2014).

[19] F. Martins, R. Genzel, D. J. Hillier, F. Eisenhauer, T. Paumard, S. Gillessen, T. Ott, and S. Trippe, Stellar and wind properties of massive stars in the central parsec of the Galaxy, Astron. Astrophys. 468, 233 (2007).

[20] G. G. Raffelt, Axion constraints from white dwarf cooling times, Phys. Lett. 166B, 402 (1986).

[21] J. Isern, E. Garcia-Berro, S. Torres, and S. Catalan, Axions and the cooling of white dwarf stars, Astrophys. J. 682, L109 (2008).

[22] J. Isern, S. Catalan, E. Garcia-Berro, and S. Torres, Axions and the white dwarf luminosity function, J. Phys. Conf. Ser. 172, 012005 (2009).

[23] J. Isern, E. Garcia-Berro, L. G. Althaus, and A. H. Corsico, Axions and the pulsation periods of variable white dwarfs revisited, Astron. Astrophys. 512, A86 (2010).

[24] M. M. M. Bertolami, B. E. Melendez, L. G. Althaus, and J. Isern, Revisiting the axion bounds from the Galactic white dwarf luminosity function, J. Cosmol. Astropart. Phys. 10 (2014) 069.

[25] J. Redondo, Solar axion flux from the axion-electron coupling, J. Cosmol. Astropart. Phys. 12 (2013) 008.

[26] N. Viaux, M. Catelan, P. B. Stetson, G. Raffelt, J. Redondo, A. A. R. Valcarce, and A. Weiss, Neutrino and Axion Bounds from the Globular Cluster M5 (NGC 5904), Phys. Rev. Lett. 111, 231301 (2013).

[27] M. Giannotti, I. Irastorza, J. Redondo, and A. Ringwald, Cool WISPs for stellar cooling excesses, J. Cosmol. Astropart. Phys. 05 (2016) 057.

[28] M. Giannotti, I. G. Irastorza, J. Redondo, A. Ringwald, and K. Saikawa, Stellar recipes for axion hunters, J. Cosmol. Astropart. Phys. 10 (2017) 010.

[29] J.-F. Fortin and K. Sinha, Constraining axion-like-particles with hard X-ray emission from magnetars, J. High Energy Phys. 06 (2018) 048.

[30] C. Dessert, A. J. Long, and B. R. Safdi, X-Ray Signatures of Axion Conversion in Magnetic White Dwarf Stars, Phys. Rev. Lett. 123, 061104 (2019).
[31] C. Dessert, J. W. Foster, and B. R. Safdi, Hard X-ray excess from the magnificent seven neutron stars, Astrophys. J. 904, 42 (2020).

[32] M. Buschmann, R. T. Co, C. Dessert, and B. R. Safdi, X-ray search for axions from nearby isolated neutron stars, arXiv:1910.04164.

[33] E. D. Carlson, Pseudoscalar conversion and X-rays from stars, Phys. Lett. B 344, 245 (1995).

[34] M. Giannotti, Fermi-LAT and NuSTAR as stellar axionscopes, in 13th Patras Workshop on Axions, WIMPs and WISPs (Verlag Deutsches Elektronen-Synchrotron, Germany, 2018), pp. 23-27.

[35] M. Meyer, M. Giannotti, A. Mirizzi, J. Conrad, and M. A. Sánchez-Conde, Fermi Large Area Telescope as a Galactic Supernovae Axionscope, Phys. Rev. Lett. 118, 011103 (2017).

[36] G. G. Raffelt, Plasmon decay into low-mass bosons in stars, Phys. Rev. D 37, 1356 (1988).

[37] B. Paxton, L. Bildsten, A. Dotter, F. Herwig, P. Lesaffre, and F. Timmes, Modules for experiments in stellar astrophysics (MESA), Astrophys. J. Suppl. Ser. 192, 3 (2011).

[38] B. Paxton, M. Cantiello, P. Arras, L. Bildsten, E. F. Brown, A. Dotter, C. Mankovich, M. H. Montgomery, D. Stello, F. X. Timmes, and R. Townsend, Modules for experiments in stellar astrophysics (MESA): Planets, oscillations, rotation, and massive stars, Astrophys. J. Suppl. Ser. 208, 4 (2013).

[39] F. Najarro, D. F. Figer, D. J. Hillier, and R. P. Kudritzki, Metallicity in the galactic center. The arches cluster, Astrophys. J. Lett. 611, L105 (2004).

[40] F. Najarro, D. F. Figer, D. J. Hillier, T. R. Geballe, and R. P. Kudritzki, Metallicity in the galactic center: The quintuplet cluster, Astrophys. J. 691, 1816 (2009).

[41] I. Hunter, D. J. Lennon, P. L. Dufton, C. Trundle, S. SimonDiaz, S. J. Smartt, R. S. I. Ryans, and C. J. Evans, The VLT-FLAMES survey of massive stars: Atmospheric parameters and rotational velocity distributions for B-type stars in the Magellanic Clouds, Astron. Astrophys. 479, 541 (2008).

[42] I. Brott et al., Rotating massive main-sequence stars II: Simulating a population of LMC early $B$-type stars as a test of rotational mixing, Astron. Astrophys. 530, A116 (2011).

[43] P. Kroupa, On the variation of the initial mass function, Mon. Not. R. Astron. Soc. 322, 231 (2001).

[44] C. Weidner and J.S. Vink, The masses, and the mass discrepancy of o-type stars, Astron. Astrophys. 524, A98 (2010).

[45] W.-R. Hamann, G. Graefener, and A. Liermann, The Galactic WN stars: Spectral analyses with line-blanketed model atmospheres versus stellar evolution models with and without rotation, Astron. Astrophys. 457, 1015 (2006).

[46] J. S. Clark, M. E. Lohr, L. R. Patrick, F. Najarro, H. Dong, and D. F. Figer, An updated stellar census of the quintuplet cluster, Astron. Astrophys. 618, A2 (2018).

[47] M. Aghakhanloo, J. W. Murphy, N. Smith, J. Parejko, M. Díaz-Rodríguez, M. R. Drout, J. H. Groh, J. Guzman, and K. G. Stassun, Inferring the parallax of Westerlund 1 from Gaia DR2, Mon. Not. R. Astron. Soc. 492, 2497 (2020).

[48] B. Davies and E. R. Beasor, The distances to star clusters hosting Red Supergiants: $\chi$ Per, NGC 7419, and Westerlund 1, Mon. Not. R. Astron. Soc. 486, L10 (2019). 
[49] J. S. Clark, B. W. Ritchie, and I. Negueruela, A VLT/ FLAMES survey for massive binaries in westerlund 1. VII. Cluster census, Astron. Astrophys. 635, A187 (2019).

[50] A. E. Piatti, E. Bica, and J. J. Claria, Fundamental parameters of the highly reddened young open clusters Westerlund 1 and 2, Astron. Astrophys. Suppl. Ser. 127, 423 (1998).

[51] G. Raffelt and L. Stodolsky, Mixing of the photon with low mass particles, Phys. Rev. D 37, 1237 (1988).

[52] Q. D. Wang, H. Dong, and C. Lang, The interplay between star formation and the nuclear environment of our Galaxy: Deep X-ray observations of the Galactic centre Arches and Quintuplet clusters, Mon. Not. R. Astron. Soc. 371, 38 (2006).

[53] R. Jansson and G. R. Farrar, A new model of the galactic magnetic field, Astrophys. J. 757, 14 (2012).

[54] R. Jansson and G. R. Farrar, The galactic magnetic field, Astrophys. J. 761, L11 (2012).

[55] J. M. Yao, R. N. Manchester, and N. Wang, A new electrondensity model for estimation of pulsar and frb distances, Astrophys. J. 835, 29 (2017).

[56] J. M. Cordes and T. J. W. Lazio, NE2001. 1. A New model for the galactic distribution of free electrons and its fluctuations, arXiv:astro-ph/0207156.

[57] M. S. Pshirkov, P. G. Tinyakov, P. P. Kronberg, and K. J. Newton-McGee, Deriving the global structure of the galactic magnetic field from faraday rotation measures of extragalactic sources, Astrophys. J. 738, 192 (2011).

[58] V. V. Izmodenov and D. B. Alexashov, Magnitude and direction of the local interstellar magnetic field inferred from Voyager 1 and 2 interstellar data and global heliospheric model, Astron. Astrophys. 633, L12 (2020).

[59] E. J. Zirnstein, J. Heerikhuisen, H. O. Funsten, G. Livadiotis, D. J. McComas, and N. V. Pogorelov, Local interstellar magnetic field determined from the interstellar boundary explorer ribbon, Astrophys. J. Lett. 818, L18 (2016).

[60] P. C. Frisch, B. G. Andersson, A. Berdyugin, H. O. Funsten, A. M. Magalhaes, D. J. McComas, V. Piirola, N. A. Schwadron, J. D. Slavin, and S. J. Wiktorowicz, Comparisons of the interstellar magnetic field directions obtained from the IBEX ribbon and interstellar polarizations, Astrophys. J. 724, 1473 (2010).

[61] M. Salvati, The local Galactic magnetic field in the direction of Geminga, Astron. Astrophys. 513, A28 (2010).

[62] K. Ferriere, Interstellar magnetic fields in the Galactic center region, Astron. Astrophys. 505, 1183 (2009).

[63] R. M. Crocker, D. I. Jones, F. Melia, J. Ott, and R. J. Protheroe, A lower limit of 50 microgauss for the magnetic field near the galactic centre, Nature (London) 463, 65 (2010).

[64] R. M. Crocker, D. I. Jones, F. Aharonian, C. J. Law, F. Melia, T. Oka, and J. Ott, Wild at heart: The particle astrophysics of the galactic centre, Mon. Not. R. Astron. Soc. 413, 763 (2011).

[65] M. Guenduez, J. B. Tjus, K. Ferrière, and R.-J. Dettmar, A novel analytical model of the magnetic field configuration in the Galactic Center, Astron. Astrophys. 644, A71 (2020).

[66] K. Mori et al., NuSTAR hard X-ray survey of the galactic center region. I. Hard X-ray morphology and spectroscopy of the diffuse emission, Astrophys. J. 814, 94 (2015).
[67] J. Hong et al., NuSTAR hard X-ray survey of the galactic center region II: X-ray point sources, Astrophys. J. 825, 132 (2016).

[68] J. K. Blackburn, in ASP Conf. Ser., Astronomical Data Analysis Software and Systems IV, edited by R. A. Shaw, H. E. Payne, and J. J. E. Hayes (ASP, San Francisco, 1999), Vol. 77.

[69] D. Porquet, J. Rodriguez, S. Corbel, P. Goldoni, R. S. Warwick, A. Goldwurm, and A. Decourchelle, Xmm-newton study of the persistent X-ray source 1e1743.1-2843 located in the galactic center direction, Astron. Astrophys. 406, 299 (2003).

[70] D. R. Wik et al., NuSTAR observations of the bullet cluster: Constraints on inverse Compton emission, Astrophys. J. 792, 48 (2014).

[71] A. Borghese et al., The multi-outburst activity of the magnetar in Westerlund I, Mon. Not. R. Astron. Soc. 484, 2931 (2019).

[72] K. K. Madsen, F. E. Christensen, W. W. Craig, K. W. Forster, B. W. Grefenstette, F. A. Harrison, H. Miyasaka, and V. Rana, Observational artifacts of NuSTAR: Ghost rays and stray light, J. Astron. Telesc. Instrum. Syst. 3, 1 (2017).

[73] H. An, K. K. Madsen, N. J. Westergaard, S. E. Boggs, F. E. Christensen, W. W. Craig, C. J. Hailey, F. A. Harrison, D. K. Stern, and W. W. Zhang, In-flight PSF calibration of the NuSTAR hard X-ray optics, Proc. SPIE Int. Soc. Opt. Eng. 9144, 91441Q (2014).

[74] G. Cowan, K. Cranmer, E. Gross, and O. Vitells, Powerconstrained limits, arXiv:1105.3166.

[75] G. Cowan, K. Cranmer, E. Gross, and O. Vitells, Asymptotic formulae for likelihood-based tests of new physics, Eur. Phys. J. C 71, 1554 (2011); Erratum, Eur. Phys. J. C 73, 2501 (2013).

[76] C. S. Reynolds, M. C. D. Marsh, H. R. Russell, A. C. Fabian, R. Smith, F. Tombesi, and S. Veilleux, Astrophysical limits on very light axion-like particles from Chandra grating spectroscopy of NGC 1275, Astrophys. J. 890, 59 (2020).

[77] M. Libanov and S. Troitsky, On the impact of magnetic-field models in galaxy clusters on constraints on axion-like particles from the lack of irregularities in high-energy spectra of astrophysical sources, Phys. Lett. B 802, 135252 (2020).

[78] A. Payez, C. Evoli, T. Fischer, M. Giannotti, A. Mirizzi, and A. Ringwald, Revisiting the SN1987A gamma-ray limit on ultralight axion-like particles, J. Cosmol. Astropart. Phys. 02 (2015) 006.

[79] G. G. Raffelt, Astrophysical methods to constrain axions and other novel particle phenomena, Phys. Rep. 198, 1 (1990).

[80] G. G. Raffelt, Astrophysical axion bounds, Lect. Notes Phys. 741, 51 (2008).

[81] J. H. Chang, R. Essig, and S. D. McDermott, Revisiting supernova 1987A constraints on dark photons, J. High Energy Phys. 01 (2017) 107.

[82] N. Bar, K. Blum, and G. D'Amico, Is there a supernova bound on axions?, Phys. Rev. D 101, 123025 (2020).

[83] M. Giannotti, B. Grefenstette, A. Mirizzi, M. Nynka, K. Perez, B. Roach, O. Straiero, and M. Xiao, Constraints on light axions from a hard $\mathrm{x}$-ray observation of betelgeuse, arXiv:2009.09059.

[84] https://github.com/bsafdi/axionSSC 